\title{
Beruflich Qualifizierte auf dem Weg ins Studium
}

\author{
Xenia Valeska Jeremias, Katja Wenger* ${ }^{*}$ Birgit Sellmer
}

\section{Zusammenfassung}

Für beruflich Qualifizierte ist der Weg in die Hochschulen und Universitäten in den letzten Jahren einfacher geworden. Die heterogenen Wege der beruflich Qualifizierten ins Studium werden wir in diesem Artikel beleuchten und aufzeigen, wie wir in unseren Mathematikvorkursen an die Vorkenntnisse unserer Studieninteressierten anknüpfen. Eine unter beruflich qualifizierten Studieninteressierten durchgeführte Befragung soll mehr Aufschluss darüber geben, über welche unterschiedlichen Wege und aus welchen persönlichen Gründen beruflich Qualifizierte ein Studium aufnehmen. Da Mathematik im Studium gerade für beruflich Qualifizierte meist eine große Hürde darstellt, hat die Technische Hochschule Wildau ihr Vorkursangebot auf die heterogener werdende Studierendenschaft angepasst.

\section{Abstract}

In recent years, the entry into universities for professionally qualified people has become more simple. This article will shed light on the heterogeneous paths of the vocationally qualified into study and show how in our mathematics pre-courses we build on the previous knowledge of our prospective students. A survey carried out among prospective professionally qualified students should provide more information about the different ways in which vocationally qualified students start their studies and for which personal reasons they do so. Because mathematics is usually a major hurdle, especially for the professionally qualified, the University of Applied Sciences Wildau has adapted its preliminary course offerings to the more heterogeneous student body.
Neben Studierenden mit schulischer Hochschulzugangsberechtigung und internationaler Bildungserfahrung bilden Studierende mit beruflicher Hochschulzugangsberechtigung einen Teil der vielfältigen Studierendenschaft der Technischen Hochschule Wildau. In diesem Beitrag werden die heterogenen Wege der beruflich Qualifizierten ins Studium beleuchtet und es wird aufgezeigt, wie in den Mathematikvorkursen an die Vorkenntnisse von Studieninteressierten angeknüpft wird bzw. wie stärker auf die unterschiedlichen Bedarfe ausgerichtete Mathematikvorkurse den Studienerfolg unterstützen können. Wir stellen folgende Fragen in den Mittelpunkt, die wir im Laufe des Beitrags erläutern wollen:

- Mit welchen unterschiedlichen Voraussetzungen, Bildungshintergründen und in welchen Lebenssituationen kommen beruflich Qualifizierte an die Hochschule?
- Was motiviert beruflich Qualifizierte dazu, ein Studium aufzunehmen?

- Wie kann in der Studienvorbereitung auf die unterschiedlichen Eingangsvoraussetzungen reagiert werden?

\section{Durchlässigkeit in Brandenburg}

Mit beruflich Qualifizierten sind nach dem Hochschulrecht Studierende gemeint, die keine schulische Hochschulzugangsberechtigung erworben haben. Häufig wird der Begriff jedoch weit gefasst ist, weil er auch beruflich qualifizierte Abiturient*innen mit Berufserfahrung einschließt (vgl. Wolter et al. 2015). Dieser weiter gefassten Definition wird in diesem Beitrag gefolgt. Die Kultusministerkonferenz empfahl im Jahr 2009 mit dem Beschluss zum Hochschulzugang für beruflich qualifizierte Bewerber*innen, die Zugangsbedingungen $\mathrm{zu}$ den Hochschulen für diejenigen ohne schulische Hochschulzugangsberechtigung zu vereinfachen.

In Brandenburg ist es bereits seit 1991 unter strengen Voraussetzungen möglich, mit beruflicher Qualifikation ein Studium aufzunehmen (Gesetz über die Hochschulen des Landes Brandenburg (Brandenburgisches Hochschulgesetz - BbgHG) 1991: § 30 Abs. 3). Bewerber*innen mussten ein Mindestalter von 24 Jahren, eine geeignete abgeschlossene Berufsausbildung und mehrjährige Berufserfahrungen nachweisen. Auch Absolvent*innen der Meisterprüfung konnten sich für das Studium bewerben. Erfüllten Studieninteressierte diese Voraussetzungen, wurden sie zu einer fachrichtungsbezogenen Eignungsprüfung zugelassen.

Die Hürden des Zugangs zur Hochschule wurden in Brandenburg im Jahr 2008 abgebaut. Interessierte, die eine bestandene Meisterprüfung oder eine gleichwertige Berechtigung nachwie- 
sen, konnten ein Studium aufnehmen. Mit einer für das beabsichtigte Studium geeigneten erfolgreich abgeschlossenen Berufsausbildung und einer anschließenden mindestens zweijährigen Berufserfahrung konnte ein Studium aufgenommen werden (vgl. Knopp \& Peine 2012). Welche Berufsausbildung für welchen Studiengang geeignet sei, wurde gesetzlich nicht näher geregelt.

Die letzte Veränderung der gesetzlichen Grundlage trat 2014 in Kraft: Bewerber*innen mit bestandener Meister- oder Technikerprüfung, mit Fortbildungsabschluss oder vergleichbaren Abschlüssen verfügen nach § 9 BbgHG über eine allgemeine Hochschulzugangsberechtigung. Ihre berufliche Qualifikation wird damit dem höchsten Schulabschluss der Bundesrepublik Deutschland gleichgestellt. Sie erhalten die uneingeschränkte Zulassung zum Studium an Universitäten und Hochschulen (Brandenburgisches Hochschulgesetz (BbgHG) 2014).

Als Hauptgründe für die politisch motivierte Durchlässigkeit der Hochschulen werden die im internationalen Ländervergleich der „Organisation for Economic Cooperation and Development" (OECD) zu geringe Akademikerquote und das durch den demographischen Wandel in Deutschland befürchtete Defizit an hochqualifizierten Fachkräften angeführt. „Die Hochschulen müssten auch deshalb neue Potenziale erschließen, weil sich die Studiennachfrage nach dem Höhepunkt der doppelten Abiturientenjahrgänge (2013) aus demographischen Gründen rückläufig entwickeln würde. Dies gelte insbesondere für Hochschulen in demographisch und ökonomisch schrumpfenden Regionen" (Wolter et al. 2014). Als ein weiteres Argument gilt die Europäisierung der Bildungspolitik. Im Bologna-Prozess wird unter "lebenslangem Lernen“ eine umfassende hochschulpolitische Strategie begriffen, Hochschulen für neue Zielgruppen zu öffnen, das Studium zu flexibilisieren und auf die spezifischen Bedürfnisse einer ver- änderten Klientel auszurichten. Die Gleichwertigkeit zwischen beruflicher und akademischer Bildung, die bis dato in Deutschland nicht gegeben war, wurde bildungspolitische Zielsetzung (vgl. Elsholz 2015).

Die politisch geforderte Gleichstellung der Bildungswege und die damit zunehmende Durchlässigkeit treffen in den Hochschulen und Universitäten teilweise auf heftigen Widerstand. Gegner dieser Veränderungen befürchten zum einen, ohne Abitur fehle die nötige Studierfähigkeit; zum anderen vermuten sie einen Ansturm auf die akademische Bildung. Weiterhin nehmen sie an, die zunehmende Vielfalt der Studierenden wirke als Gegenpol einer „Elitenausbildung“ (vgl. Wolter et al. 2014; Hanft 2015; Seidel 2014).

Die Erfahrungen mit beruflich Qualifizierten an der TH Wildau in Beratung und Vorbereitungskursen zeigen, dass dies eine Gruppe mit besonderen Bedarfen ist, aber auch mit besonderen Kompetenzen. Zu diesen zählen ein hohes $\mathrm{Maß}$ an Berufserfahrung, praktische Kompetenzen, eine häufig im Berufsleben erwachsene Motivation, ein Studium aufzunehmen, und damit eine starke Zielorientierung. Im Folgenden wird eine Befragung beruflich qualifizierter Studieninteressierter dargestellt, die an der TH Wildau im Rahmen eines Beratungsangebots durchgeführt wurde. Sie soll einen Eindruck von den Lebenslagen beruflich qualifizierter Studieninteressierter vermitteln und die Motivlagen zur Aufnahme eines Studiums in den Vordergrund rücken.

\section{Beruflich qualifizierte Studien- interessierte an der TH Wildau}

Insgesamt gab es im Bundesgebiet in den zurückliegenden zwei Jahrzehnten einen Wachstumstrend bei Studienanfänger ${ }^{*} i n n e n$ ohne Allgemeine Hochschulreife und ohne Fachhochschulreife. Ihr bundesweiter Anteil an allen Studierenden im ersten Semester erhöhte sich von $0,6 \%$ im Jahr 1997 auf 2,8 \% im Jahr 2014. Ins- gesamt hält sich die Quote beruflich Qualifizierter jedoch bundesweit unter $3 \%$. Gleichwohl können drei Ballungszentren in Deutschland einen höheren Anteil an Studienanfänger ${ }^{*} i n n e n$ ohne Allgemeine Hochschulreife und ohne Fachhochschulreife nachweisen: Hamburg (4,47\%), Nordrhein-Westfalen $(4,28 \%)$ und Berlin (3,42 \%) (vgl. Nickel \& Schulz 2017). Die hohe Siedlungsdichte in diesen urbanen Räumen, aber auch Strategien in Bezug auf beruflich Qualifizierte haben vermutlich zu diesem Anstieg geführt. In Hamburg und Berlin wurden beispielsweise Vorabquoten bei der Studienplatzvergabe in Höhe von 3-10 \% für Studienbewerber*innen ohne schulische Hochschulzugangsberechtigung eingeführt.

Im Rahmen der Beratung des Projekts "Studienstart 2.0 // Mobile Beratung für beruflich Qualifizierte“1 nahmen im akademischen Jahr 2016/2017 insgesamt 51 Personen (davon 12 weiblich und 39 männlich) an einer Befragung teil. Die Ergebnisse der Umfrage sind nicht repräsentativ.

Zunächst erfassten wir soziographische Daten, um die soziale Heterogenität zu bestimmen. Unter denen, die das Beratungsangebot "Studienstart 2.0" in Anspruch nahmen, waren deutlich mehr männliche Studieninteressierte, nämlich $76 \%$. Im Vergleich zum Geschlechterverhältnis der Gesamtstudierendenzahlen (Verteilung der Studierenden 2016/17: männlich 3.786, weiblich 1.384; das entspricht männlich $63 \%$ und weiblich $37 \%$ ) sind also deutlich mehr männliche Ratsuchende an einem Studium aus dem Beruf heraus interessiert. Der Anteil der Männer liegt bei nicht traditionellen Studierenden auch nach der Untersuchung von Wolter et al. (2015) über jenem in der Gesamtgruppe der Studierenden. So waren $55 \%$ der nicht traditionellen Studienanfänger ${ }^{*} i n n e n$ im Studienjahr 2013 männlich. Warum der Anteil der Männer höher ist als der Frauenanteil, ist bisher noch unklar (vgl. Wolter et al. 2015).

Dass beruflich qualifizierte Studieninteressierte in der Kategorie Alter über dem Durchschnitt der Studierenden 
insgesamt (23,9 Jahre [vgl. Middendorff et al. 2013]) liegen, ist nicht verwunderlich (siehe Abb. 1). Bemerkenswert ist aber die Erkenntnis, dass $41 \%$ der Befragten zwischen 25 und 31 Jahren und $24 \%$ zwischen 32 und 38 Jahren alt sind. Demnach strebt der größte Teil beruflich Qualifizierter erst nach längerer Berufstätigkeit ein Studium an. Dies impliziert einen mehrjährigen Abstand zum letzten Schulbesuch und verweist damit auf einen speziellen Bedarf an Angeboten für den Einstieg ins Studium. Insbesondere das Auffrischen schulischer Mathematikkenntnisse ist bei diesen Rahmenbedingungen häufig notwendig.

Studienanfänger*innen über 25 Jahre befinden sich üblicherweise unmittelbar vor bzw. in der Phase der Familienplanung und sind im Alter von 32 und 38 Jahren wahrscheinlich in Familienverantwortung gebunden. So stehen sie mit dem Start in das Studium vor der Herausforderung, das Studium mit der Familie und häufig auch mit dem Beruf zu vereinbaren. Die beruflich qualifizierten Studieninteressierten leben zu 19,6 \% mit Kindern. Bei traditionell Studierenden studieren hingegen nur $5 \%$ mit Kindern (vgl. Middendorff et al. 2013).

Für ein Studium bis 2 Jahre nach der Berufsausbildung entscheiden sich $34 \%$ der Befragten. Das sind diejenigen, die über eine schulische Hochschulzugangsberechtigung verfügen. Am häufigsten nehmen die beruflich qualifizierten Studierenden nach 5-10 Jahren ein Studium auf. Insgesamt wird deutlich, dass sie häufig viele Jahre berufstätig sind, bevor sie sich für ein Studium entscheiden (siehe Abb. 2).

Über 70 \% der befragten Studieninteressierten nutzen ihre berufliche Qualifikation, d. h. die Berufsausbildung und zwei Jahre Berufserfahrung, als Hochschulzugangsberechtigung bzw. den Meister- oder Technikerabschluss, um ein Studium aufnehmen. Etwa $30 \%$ haben vor der Berufstätigkeit eine schulische Hochschulzugangsberechtigung, also das Abitur oder die Fachhochschulreife, erworben (siehe Abb. 3).

Das Bild der Befragten wird abgerundet durch die Frage, welche Berufsausbildungen die Studieninteressierten mit beruflicher Qualifikation zuvor abgeschlossen hatten. Am häufigsten (11 von 51) wurde eine Ausbildung zur

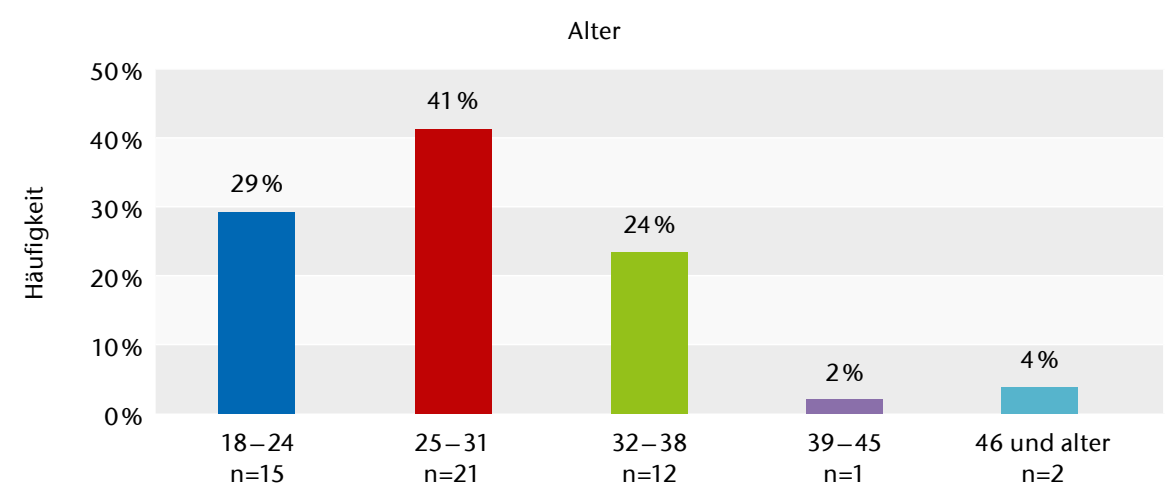

Abb. 1) Alter der befragten Studieninteressierten mit beruflicher Qualifikation, $n=51$ (eigene Darstellung).

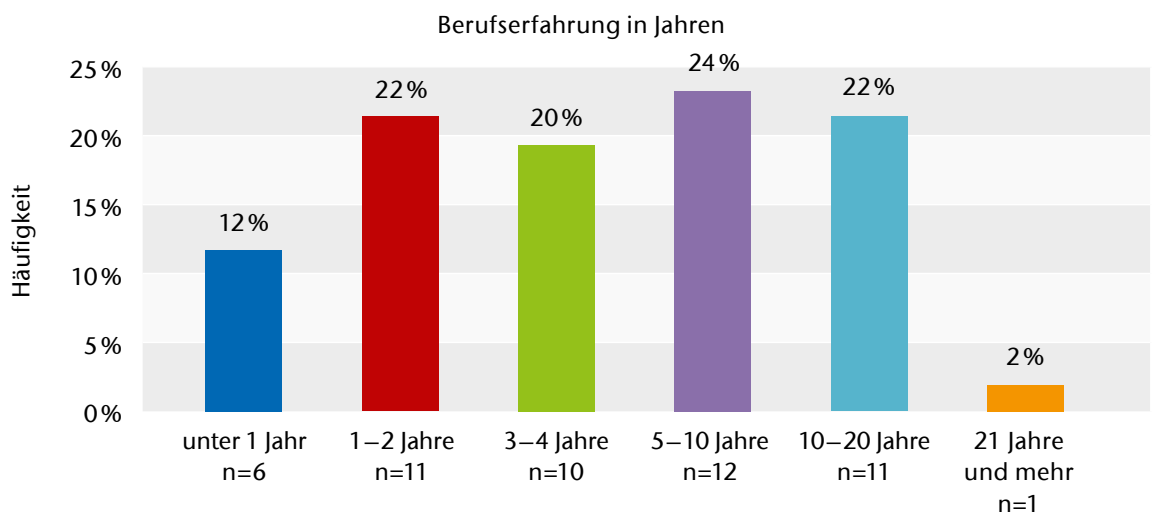

Abb. 2) Berufserfahrung der befragten Studieninteressierten mit beruflicher Qualifikation, $n=51$ (eigene Darstellung).

Hochschulzugangsberechtigung

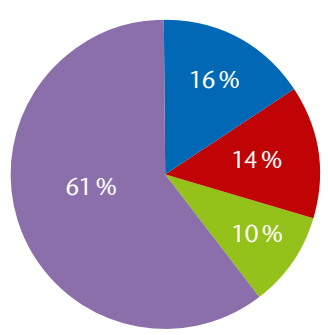

Abitur

Fachhochschulreife Meister/Techniker

Berufsausbildung und 2 Jahre Berufserfahrung

Abb. 3) Hochschulzugangsberechtigung der befragten Studieninteressierten mit beruflicher Qualifikation, $n=51$ (eigene Darstellung).

Kauffrau/zum Kaufmann beendet, gefolgt von Mechaniker*innen (9 von 51) und Elektriker*innen (9 von 51).

Kernelement der Befragung war die Studienmotivation, die in einer offenen Frage erhoben wurde. Dies ist vor allem auch deswegen relevant, weil fehlende Motivation als einer der drei Hauptgründe für den Studienabbruch genannt wird (vgl. Heublein et al. 2009). Aus den Antworten auf die offene Frage „Was motiviert Sie, ein Studium aufzunehmen?" wurden nach dem Konzept der Inhaltsanalyse vier
Motivkategorien (siehe Abb. 4) abgeleitet (vgl. Mayring 2010).

\section{Motiv „beruflich aufsteigen“}

Über das Studium eine bessere Aussicht auf dem Arbeitsmarkt allgemein zu erlangen oder ganz konkret im aktuellen Unternehmen eine bestimmte höhere Position einnehmen zu können, dies erhoffen sich die meisten Studieninteressierten mit beruflicher Qualifikation. Im Mittelpunkt dieses Motivs stehen bessere Aussichten für die Zukunft und die Änderung der 
beruflichen Stellung in einem Unternehmen. Wie die beruflichen Perspektiven aussehen sollen, bleibt bei vielen eher unkonkret. Es wird aber ein Änderungswunsch deutlich: „bessere berufliche Möglichkeiten“, "angenehmere Arbeit“, „bessere Stellung auf dem Arbeitsmarkt“, „bessere Arbeitsplätze“ und "berufliches Vorankommen" sind einige Beispielaussagen der Befragten. Teilweise wird ein Handlungs- und Veränderungsdruck als dahinterliegender Motivator beschrieben: ,jetziger Stand nicht bis Rente machbar (körperlich)“. Mit der Veränderung der beruflichen Perspektiven verbinden die Befragten den Wunsch, beruflich aufzusteigen: "berufliche Aufstiegschancen“, „evtl. eine Führungsposition“, "größere Aufstiegschancen". Dabei werden nur vereinzelt berufliche Perspektiven explizit benannt: „Prüfingenieur bei der technischen Untersuchung werden“ bzw. „Chance auf eine Fachtechniker-Stelle“.

\section{Motiv „Sich beruflich weiterbilden“} Im Mittelpunkt dieses Motivs steht die berufliche (Weiter-)Bildung bzw. die Veränderung der eigenen Person durch das Erlernen theoretischen und praktischen Wissens im Studium. Diese Studieninteressierten wollen ihre fachlichen Kenntnisse erweitern. Das Studium wird als Weiterbildungsoption in der beruflichen Entwicklung gesehen. Folgende Beispielaussagen machen dies deutlich: „berufliche und fachliche Weiterbildung“, „Weiterbildung + Qualifizierung“. Es geht neben dem beruflich-fachlichen auch allgemein um den Ausbau des Wissenstandes: „neues Wissen erlernen“, „viel neues Wissen“, „Interessen an Bildung“, "Wissensaufbau" sowie "Wunsch nach mehr Wissen \& Erfahrung“.

\section{Motiv "Herausforderungen suchen“}

Beruflich Qualifizierte suchen durch ein Studium neue Herausforderungen, "Gehirnfutter" und eine fordernde Tätigkeit. Die aktuelle Beschäftigung ist nicht in gewünschtem Maße anspruchsvoll, sodass ein Bedürfnis nach Herausforderungen als Motivlage entsteht: „mehr gefordert werden“, „neue Herausforderungen“, "Gehirnfutter“, „anschließend eine anspruchsvolle Tätigkeit ausüben“ sowie „arbeiten auf technisch höherem Niveau“.

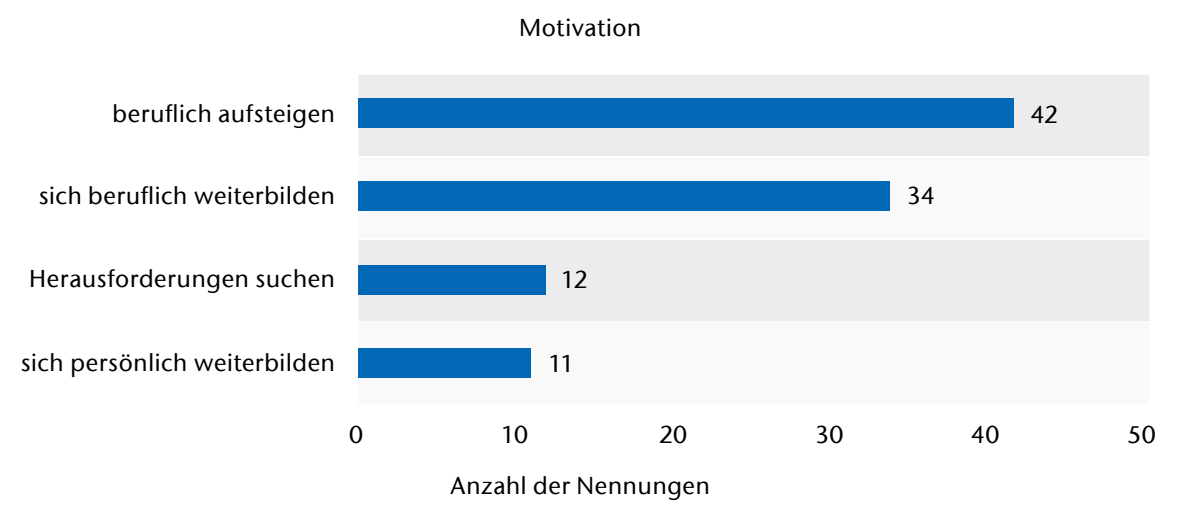

Abb. 4) Motivation der befragten Studieninteressierten, Mehrfachnennungen, $n=51$ (eigene Darstellung)

Motiv „sich persönlich weiterbilden“ Beim Motiv „sich persönlich weiterbilden“ steht die Person im Mittelpunkt. Als relativ allgemeines persönliches Ziel nennen die Befragten „persönliche Weiterbildung“ und „persönliche Weiterentwicklung“. Einige der zukünftigen Studierenden mit beruflicher Qualifikation nennen ganz konkret das Ziel "Selbstverwirklichung“. Das Studium wird als Möglichkeit gesehen, die eigenen Fähigkeiten und Talente zu nutzen, um die Persönlichkeit zu entfalten.

Die Beschreibung der Motivlagen beruflich qualifizierter Studieninteressierter zeigt deutlich die hohe Motivation und die Zielorientierung bei der Studienorientierung und kurz vor Aufnahme des Studiums (vgl. auch Burchard 2017). In Verbindung mit den Kompetenzen, die die beruflich Qualifizierten aus ihrer Berufserfahrung einbringen, kann die Ausgangslage dieser Zielgruppe im Vergleich zu traditionell Studierenden als vergleichsweise positiv angesehen werden. Insbesondere lässt sich aus den Motiven "beruflich weiterbilden“ und "beruflich aufsteigen" ein zielgerichtetes und beharrliches Hinarbeiten auf den Studienabschluss ableiten. Außerdem kann angenommen werden, dass der Wunsch nach kognitiven Herausforderungen zu der Bereitschaft führt, sich intensiv mit Studieninhalten auseinanderzusetzen. Demgegenüber bedeutet die längere Berufstätigkeit vor Aufnahme des Studiums, dass diese Studierenden in Modulen mit einem Theorieschwerpunkt (z. B. Mathematik) stärkere Unterstützung benötigen. Auf beide Aspekte sollte bei der Gestaltung der Studienvorbereitung verstärkt eingegangen werden.
Die TH Wildau bietet bereits Studiengänge an, die mit einer Berufstätigkeit kombiniert werden können. In diesen Studiengängen ist in allen Modulen die Ergänzung durch Online-Lernmaterialien vorgesehen, sodass auf das Bedürfnis der Berufstätigen nach zeitund ortsunabhängigem Lernen eingegangen wird. Zudem bietet die Hochschule für alle Studienanfänger*innen in der Studieneingangsphase verschiedene Wege, um den Übergang ins Studium gut vorzubereiten. So können beruflich Qualifizierte an einem Vorsemester teilnehmen und Studienvorbereitungskurse belegen. Auch das Beratungsangebot im Studium umfasst mit individueller Mathematik- oder Lernberatung sowie mit dem Studiencoaching Möglichkeiten, sich persönlich beim Studienstart begleiten zu lassen. Ein Beispiel für eine bereits erprobte Umsetzung eines differenzierten Angebots für diese Zielgruppe stellen die Vorbereitungskurse für Mathematik dar. Dieses Konzept soll daher im Folgenden genauer beschrieben werden. In der Studienorganisation und in der Angebotsstruktur wäre darüber hinaus jedoch noch eine Reihe von Veränderungen und Innovationen denkbar, um an die Potenziale der beruflich Qualifizierten noch besser anknüpfen zu können. Darauf wird im letzten Abschnitt eingegangen.

\section{Studienvorbereitung in Mathematik}

Da Mathematik im Studium für viele Studierende eine große Hürde darstellt, hat die TH Wildau ihr Vorkursangebot in diesem Fach auf die heterogener werdende Studierendenschaft angepasst. Hochschulen setzen sich schon seit längerem mit der heterogener werdenden Studierendenschaft 
auseinander (vgl. Hanft 2015). Betrachtet man die Studierendenschaft unter Berücksichtigung verschiedener Heterogenitätsdimensionen (soziodemographische Merkmale, Motivation etc.), wird klar, dass beruflich Qualifizierte nur einen kleinen Teil einer insgesamt heterogenen Gruppe ausmachen. Als Folge der größer werdenden Heterogenität wurden bildungspolitisch verschiedene Maßnahmen initiiert, welche sich hauptsächlich auf die Gestaltung der Studieneingangsphase konzentrieren. Beispielsweise wurden bereits in den 1980er Jahren erste Mathematikvorkurse eingerichtet (vgl. Abel \& Weber 2014). Aktuell zielen mehr als zwei Drittel der im Qualitätspakt Lehre geförderten Hochschulprojekte auf eine Verbesserung der Studieneingangsphase ab. Dabei wird Heterogenität vielfach als Auslöser für abnehmende Studierfähigkeit angesehen. Dem soll mit verschiedenen Maßnahmen wie Beratungsangeboten, Brückenkursen oder Tutorien begegnet werden (vgl. Hanft 2015).

Im Folgenden werden die Ausgangssituation und der aktuelle Stand der Vorkurse an der TH Wildau beschrieben.

\section{Ausgangssituation und Vorüberlegungen}

Bis ins Jahr 2012 gab es zwei Mathematikvorkurse, einen für angehende Wirtschaftsstudierende und einen für angehende Ingenieurstudierende, die fünf bzw. sechs Tage dauerten. Gedanklicher Ausgangspunkt dieser Kurse war der "klassische“ Bildungsweg, bei dem die Teilnehmenden nach Abitur und evtl. einem Jahr Unterbrechung ein Studium aufnehmen und daher eine gewisse Auffrischung der Mathematikkenntnisse, aber auch nicht mehr, benötigen. In diesen Kursen wurde der Mathematikstoff der Mittel- und Oberstufe überblicksartig behandelt. Umfangreiche betreute Übungsanteile waren nicht integriert. Solche Übungsanteile sind aber essentiell, um sich die mathematischen Inhalte nachhaltig erschließen zu können, da nur durch Üben und Wiederholen eine fundierte Auseinander- setzung mit den Inhalten stattfindet. Dies trifft insbesondere auf angehende Studierende, die nicht den "klassischen" Bildungsweg beschritten haben, zu. Da beruflich Qualifizierte die Schule mindestens fünf Jahre vor Studienbeginn abgeschlossen haben müssen, liegt der letzte Mathematikunterricht notwendigerweise länger zurück. Wissen und Fähigkeiten, die nicht regelmäßig genutzt werden, gehen aber schnell verloren, sodass die Aktivierung von nötigem Vorwissen für das Studium bei dieser Zielgruppe einen höheren Aufwand bedeutet. Dies gilt vor allem, weil Vorwissen, an das beim Lernen angeknüpft werden kann, einer der wichtigsten Einflussfaktoren für erfolgreiches Lernen ist (vgl. Krause \& Stark 2006). Ein letzter Aspekt, der die Notwendigkeit einer ausführlichen Mathematikvorbereitung begründet, ist, dass beruflich Qualifizierte keine gymnasiale Oberstufe durchlaufen haben, bestimmte Themen (wie Differenzial-, Integralund Vektorrechnung) ihnen also komplett unbekannt sind. Hier muss das Vorwissen, an das andere anknüpfen können, im Studienvorbereitungskurs erst aufgebaut werden.

\section{Anpassung des Vorkursangebots}

Um diesen neuen Zielgruppen besser gerecht zu werden, wurde u. a. im Rahmen des Qualitätspakt-Projekts "SOS - Strukturierung und Optimierung des Selbststudiums " 2 das Vorbereitungsangebot breiter aufgestellt: Es werden nunmehr sechs unterschiedlich lange Mathematikvorkurse angeboten. Statt sich an den gewünschten Studienrichtungen zu orientieren, liegt der Fokus nun auf den unterschiedlichen Niveaus der Vorkenntnisse, die die zukünftigen Studierenden mitbringen. ${ }^{3}$ Zudem wird auf die zeitlichen Möglichkeiten der angehenden Studierenden, die teilweise neben Studienvorbereitung bzw. Studium arbeiten oder Betreuungsverpflichtungen haben, Rücksicht genommen. Die Inhalte, die in den verschiedenen Kursen angeboten werden, gleichen sich; Unterschiede bestehen in der Dauer bzw.
Intensität, mit der diese Inhalte behandelt werden, sowie in der Lehr-/Lernform. So werden einige beispielsweise im Blended-Learning-Format, also als Präsenzkurs mit integrierten OnlinePhasen, angeboten. Ein Online-Test, der auf der Hochschul-Website veröffentlicht ist, dient als Entscheidungshilfe, welcher Kurs der passende ist.

Im Folgenden werden die nun angebotenen Kurse beschrieben:

- Mathematikmodul im Vorsemester: Im Rahmen des Vorsemesters des TH-College (https://www.thwildau.de/vorsemester) ist Mathematik ein Kernmodul. Es umfasst ähnliche Inhalte, wie sie nachfolgend für den Mathematik-Intensivkurs beschrieben werden.

Nach der Pilotphase im Projekt SOS wurde das breitere Vorkursangebot verstetigt, sodass es nun - wie auch die Vorkurse in anderen Fächern - vom Technologietransfer- und Weiterbildungszentrum e. V. an der TH Wildau (TWZ e. V., wWw.th-wildau.de/studienvorbereitungskurse) kostenpflichtig angeboten wird.

- Mathematik-Intensivkurs, werktags und Mathematik-Intensivkurs, samstags: Diese Kurse im Blended-Learning-Format wurden speziell mit dem Fokus auf nicht traditionell Studierende eingerichtet. Während der vier Kurswochen wechseln sich Präsenzaktivitäten (Vorlesungen und Tutorien) mit strukturierten Online-Phasen ab. Der Vorteil dieser Lehr-/Lernform ist - neben der Hinführung auf studentische Lerntechniken - die individuelle Anpassung des Übungsaufwands an die eigenen Bedürfnisse gerade in den Online-Phasen, die von fachlich kompetenten Ansprechpersonen betreut werden. Außerdem erhöht sich dadurch die Vielfalt der Methoden, mit denen die mathematischen Inhalte vermittelt werden, sodass unterschiedlichen kognitiven Herangehensweisen

\footnotetext{
${ }^{2}$ Gefördert vom BMBF unter dem Förderkennzeichen 01PL11042 im Rahmen des Qualitätspakts Lehre.

${ }^{3}$ Dies ist auch deswegen sinnvoll, weil diejenigen, die das Studium direkt nach der Schule beginnen, dort ebenfalls keine fachspezifische Mathematik gelernt haben.
} 
Rechnung getragen wird. Aber auch während der Präsenzphasen wird viel Wert auf umfangreiche Übungen gelegt. Da der Anteil berufsbegleitend Studierender an der TH Wildau recht hoch ist und diese Studierenden unter der Woche arbeiten gehen, muss es eine Variante des Kurses geben, die am Samstag stattfindet, um auch dieser Zielgruppe eine Mathematikvorbereitung zu ermöglichen. Das ist insbesondere deswegen wichtig, weil berufsbegleitend Studierende ja bereits einen Beruf erlernt haben und deswegen nicht direkt von der Schule kommen können. Der Aktivierungsaufwand für das nötige Vorwissen ist daher entsprechend höher.

- Mathematik-Crashkurs: Dieser Kurs entspricht (bis auf die nicht mehr vorhandene Aufteilung nach Studiengängen) den bereits vor 2012 bestehenden Vorkursen. Er zielt auf Studienanfänger*innen, die direkt von der Schule kommen bzw. nur eine kurze Unterbrechung des Lernweges hatten, sodass die Aktivierung des nötigen Wissens üblicherweise unkompliziert und mit wenig Aufwand zu bewerkstelligen ist.

Eine Teilnehmerin des Mathematik-Intensivkurses 2017 hatte ihren mittleren Schulabschluss im Jahr 1992 gemacht. Die Differenzialrechnung war für sie völlig neu, da dies ein mathematischer Inhalt der Oberstufe ist. Nach der entsprechenden Vorlesung erklärte sie auf Nachfrage, sie sei vom Verstehen noch so weit entfernt, dass sie nicht mal Fragen zu diesem Thema stellen könne. Durch intensives Arbeiten und Üben in der Online-Phase schaffte sie es innerhalb von 1,5 Tagen, $100 \%$ der Punkte in einem OnlineTest zu einfachen Ableitungen zu erreichen!
- Mathematik-Last-Minute-Kurs, vormittags und Mathematik-LastMinute-Kurs, nachmittags: Da erfahrungsgemäß nicht alle Studierenden, deren Vorkenntnisse einer Auffrischung bedürfen, tatsächlich an einem Vorbereitungskurs vor Studienbeginn teilnehmen ${ }^{4}$, gibt es in diesen Kursen, die an den ersten drei Samstagen des Semesters stattfinden, eine weitere Möglichkeit, sich benötigte Mathematikfähigkeiten anzueignen. Die Kurse werden von Studierenden durchgeführt und gleichen daher einem Tutorium, in dem viel anhand von Fragen der Teilnehmenden gearbeitet wird. Im Laufe des Kurses vermischen sich die klassischen Vorkursinhalte mit den Inhalten des Semesters, da diejenigen, die Defizite beim Vorwissen haben, üblicherweise auch mit den Semesterinhalten Schwierigkeiten haben.

Ein Teilnehmer des MathematikIntensivkurses 2016 hatte seinen mittleren Schulabschluss im Jahr 1987 gemacht. Es war - gerade zu Beginn - deutlich zu erkennen, dass ihm die mathematischen Inhalte, die im Vorkurs durchgearbeitet wurden, große Schwierigkeiten bereiteten. Im Laufe der vier Wochen des Kurses konnten diese Schwierigkeiten zwar nicht ausgeräumt, doch aber immerhin gemindert werden, was sich $u$. a. darin zeigte, dass er begann, Fragen zu stellen. Durch den Besuch diverser Tutorien im Semester schaffte er es, die Mathematik I-Klausur im ersten Versuch zu bestehen!

Aus unserer Erfahrung sind das Mathematikmodul im Vorsemester sowie die Mathematik-Intensivkurse besonders empfehlenswert für beruflich qualifizierte Studienanfänger*innen, da sie nicht (nur) wochentags, sondern auch am Wochenende angeboten werden, eine intensive, länger andauernde Beschäftigung mit der Mathematik ermöglichen und verschiedene Lernformen (z. B. durch das Blended-Learning-Format) unterstützen.

Flankiert wird dieses neue Vorkurssystem durch Unterstützungsangebote im Studium, die ebenfalls vom Projekt SOS initiiert wurden: So wurde in den letzten Jahren ein Tutoriensystem aufgebaut, das Studierenden aller Studiengänge die Möglichkeit bietet, im Semester niedrigschwellig Hilfe in Mathematik zu erhalten. Zudem wird seit WiSe 15/16 eine Mathematikberatung angeboten, die beispielsweise zu der Frage berät, wie die Studienvorbereitung am besten gelingen kann oder wie die Vorbereitung auf eine Nachprüfung gestaltet werden sollte. Dieses Beratungsangebot ist eng mit den weiteren Beratungsangeboten der Hochschule verzahnt.

\section{Fazit und Ausblick}

Die TH Wildau hat in den vergangenen Jahren - insbesondere bei Beratung und Studienvorbereitung - viel getan, um den Bedürfnissen der heterogener werdenden Studierendenschaft Rechnung zu tragen. Inwieweit diese Maßnahmen den Studienerfolg beeinflussen, wird sich erst in einigen Jahren zeigen. Bislang stehen alle Studierenden, die an der Beratung "Studienstart 2.0" und den veränderten Vorkursen teilgenommen haben, erst am Beginn bzw. in der Mitte ihres Studiums. Folgt man Hanft (2015), sind diese Angebote ein guter erster Schritt, dem allerdings weitere folgen sollten. Auch die Berücksichtigung der organisationalen Ebene im Studium ist demzufolge notwendig, damit verschiedenste Gruppen ihr Studium erfolgreich absolvieren können.

Die Befragung der beruflich qualifizierten Studieninteressierten an der TH Wildau konnte zeigen, dass diese Gruppe der Studierenden besondere Bedarfe hat, aber auch Ressourcen durch Berufserfahrung und eine zielgerichtete Studienmotivation einbringt. Die Kenntnis der Motivlagen der beruflich Qualifizierten wird z. B. für die Studierendengewinnung ge- 
nutzt. Passgenaue Angebote im Sinne eines Weiterbildungs- bzw. Studienangebots, die sich an den Bedarfen und Stärken ausrichten, können den Anteil der beruflich Qualifizierten an den Studieninteressierten signifikant erhöhen. Im Folgenden wird daher für eine Flexibilisierung des Studiums und eine innovative Gestaltung der Module plädiert. Die TH Wildau ist hier mit dem Teilzeitstudium bereits einen Schritt gegangen, da ein Teilzeitstudium leichter mit beruflichen und familiären Verpflichtungen zu vereinen ist.

Um den Zugang zum Studium niedrigschwelliger zu gestalten, würden sich Zertifikatsmodule anbieten, die auf ein (späteres) Studium anrechenbar sind. So könnten die Motive „sich beruflich weiterbilden“ und "Herausforderungen suchen" eine Entsprechung im Angebot finden. Für das Motiv „sich persönlich weiterbilden“ könnten ebenfalls entsprechende Zertifikatsmodule aufgelegt werden, beispielsweise aus dem Bereich der Personalentwicklung. Dadurch wäre es möglich, flexibel ein Studium auszuprobieren, bevor eventuell die Berufstätigkeit aufgegeben wird und damit die Lebensplanung umgestellt werden muss.

Sowohl bei der Entwicklung der Studiencurricula als auch bei der Modulgestaltung sollte stärker an die Erfahrungen und Kompetenzen aus den Berufsfeldern angeknüpft werden, beispielsweise dadurch, dass im ersten Semester Module enthalten sind, die für beruflich Qualifizierte eine Brücke zu ihren Berufserfahrungen herstellen. Dies würde auch den übrigen Studierenden helfen, die - gerade in den Ingenieurstudiengängen - vielfach über Schwierigkeiten mit den theoretischen Grundlagenmodulen in den ersten Semestern berichten. Insgesamt ist ein praxisbezogener Einblick in das Studienfach zu Beginn günstig für die Motivation aller Anfänger*innen.

Schließlich sollte geprüft werden, ob es Sinn macht, analog zu dem Vorbereitungsangebot für Mathematik weitere passgenaue Angebote, insbesondere für theoriebasierte "Wissensmodule“, zu entwickeln. Die zunehmende Heterogenität der Studierenden macht es vor dem Hintergrund der gewünschten Durchlässigkeit wahrscheinlich, dass „nullte“ Semester und ähnliche
Angebote für einen fließenden Übergang ins Studium zum Standard an den Hochschulen werden.

\section{LITERATUR}

Abel H, Weber B (2014) 28 Jahre Esslinger Modell Studienanfänger und Mathematik. In: Bausch I, Biehler R, Bruder R, Fischer PR, et al. (eds) Mathematische Vor- und Brückenkurse. Konzepte, Probleme und Perspektiven. Konzepte und Studien zur Hochschuldidaktik und Lehrerbildung Mathematik. Springer Spektrum, Wiesbaden, ISBN: 978-3-658-03064-3, pp 9-19. doi: 10.1007/978-3-658-03065-0_2

Banscherus U, Kamm C, Otto A (2015) Information, Beratung und Unterstützung von nicht-traditionellen Studierenden. Angebote der Hochschulen und deren Bewertung durch die Zielgruppe. In: Hanft A, ZawackiRichter O, Gierke WB (eds) Herausforderung Heterogenität beim Übergang in die Hochschule. Waxmann, Münster New York, NY, ISBN: 978-3-8309-3100-3, pp 81-96

(2014) Brandenburgisches Hochschulgesetz (BbgHG).

Burchard A (2017) Auch ohne Abitur an der Uni erfolgreich. Nicht-traditionelle Studierende haben kaum schlechtere Noten als klassische, brechen aber öfter ab. http://www.tagesspiegel.de/wissen/studierenohne-abitur-auch-ohne-abitur-an-der-uni-erfolgreich/20735028.html. Accessed 09 Jan 2018

Elsholz U (2015) Überwindung der Trennung zwischen beruflicher und akademischer Bildung? Bildungstheoretische, bildungspolitische und didaktische Herausforderungen. In: Elsholz U (ed) Beruflich Qualifizierte im Studium. Analysen und Konzepte zum Dritten Bildungsweg, 1st edn. wbv, Bielefeld, ISBN: 3763956050, pp 245-260

(1991) Gesetz über die Hochschulen des Landes Brandenburg (Brandenburgisches Hochschulgesetz BbgHG).

(2008) Gesetz über die Hochschulen des Landes Brandenburg (Brandenburgisches Hochschulgesetz BbgHG).

Hanft A (2015) Heterogene Studierende - homogene Studienstrukturen. In: Hanft A, Zawacki-Richter O, Gierke WB (eds) Herausforderung Heterogenität beim Übergang in die Hochschule. Waxmann, Münster, New York, NY, ISBN: 978-3-8309-3100-3, pp 13-28

Heublein U, Hutzsch C, Schreiber J, Sommer D, Besuch G (2009) Ursachen des Studienabbruchs in Bachelorund in herkömmlichen Studiengängen. Ergebnisse einer bundesweiten Befragung von Exmatrikulierten des Studienjahres 2007/08. HIS: Projektbericht

Knopp L, Peine F-J (eds) (2012) Brandenburgisches Hochschulgesetz. Handkommentar, 2 nd edn. Nomos, Baden-Baden. ISBN: 978-3-8329-7324-7

Krause U-M, Stark R (2006) Vorwissen aktivieren. In: Mandl H, Friedrich HF (eds) Handbuch Lernstrategien. Hogrefe, Göttingen, ISBN: 3801718131, pp 38-49

Mayring P (2010) Qualitative Inhaltsanalyse. Grundlagen und Techniken, 11., aktualisierte und überarb. Aufl. Pädagogik. Beltz, Weinheim, Basel. ISBN: 9783407255334

Middendorff E, Apolinarski B, Poskowsky J, Kandulla M Netz N, Naumann H, Buck D (2013) Die wirtschaftliche und soziale Lage der Studierenden in Deutschland 2012. 20. Sozialerhebung des Deutschen Studentenwerks durchgeführt durch das HIS-Institut für Hochschulforschung

Nickel S, Schulz N (2017) Update 2017: Studieren ohne Abitur in Deutschland Überblick über aktuelle Entwicklungen, CHE AP 195. CHE Arbeitspapier. Centrum für Hochschulentwicklung $\mathrm{gGmbH}$, Gütersloh. ISBN: 978-3-941927-80-3
Seidel S (2014) Defizitär oder produktiv: Die Heterogenität der Studierenden. In: Seidel S, Wielepp F (eds) Diverses. Heterogenität an der Hochschule. Institut $f$. Hochschulforschung Wittenberg, Lutherstadt Wittenberg, ISBN: 978-3-937573-42-7, pp 6-21

Wolter A, Banscherus U, Kamm C, Otto A, Spexard A (2014) Durchlässigkeit zwischen beruflicher und akademischer Bildung als mehrstufiges Konzept. Bilanz und Perspektiven. Beiträge zur Hochschulforschung(4):8-39

Wolter A, Dahm G, Kamm C, Kerst C, Otto A (2015) Nicht-traditionell Studierende in Deutschland - Werdegänge und Studienmotivation. In: Elsholz U (ed) Beruflich Qualifizierte im Studium. Analysen und Konzepte zum Dritten Bildungsweg, 1st edn. wbv, Bielefeld, ISBN: 3763956050, pp 11-34

\section{AUTOREN}

Dr. Xenia Valeska Jeremias

Katja Wenger

Technische Hochschule Wildau

E-Mail für Korrespondenz:

katja.wenger@th-wildau.de

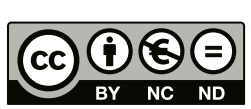

